\title{
NANOPARTICLE-BASED FRET PAIR SELECTION FOR INVESTIGATION OF METALLOTHIONEIN DIMERIZATION
}

\author{
${ }^{1}$ Kristyna PAVELICOVA, ${ }^{1,2}$ Lucie VANICKOVA, ${ }^{1}$ Marcela VLCNOVSKA, \\ 1,2Marketa VACULOVICOVA \\ ${ }^{1}$ Department of Chemistry and Biochemistry, Mendel University in Brno, Czech Republic, EU \\ k.pavelicova@seznam.cz
}

${ }^{2}$ Central European Institute of Technology, Brno University of Technology, Brno, Czech Republic, EU

https://doi.org/10.37904/nanocon.2019.8566

\begin{abstract}
Metalloproteins (MTs) are one of the most diverse classes of proteins, with the intrinsic metal atoms providing a catalytic, regulatory and structural role crucial to protein functioning. When stored under aerobic conditions, MTs might form dimers (as well as higher oligomers) that play an essential role as mediators in oxidoreduction signalling pathways. In this work, we explore the non-oxidative dimerization process characterized by metal (Cd) bridging of MT monomers by Förster resonance energy transfer. The formation of MT dimers/oligomers was investigated by applying capillary electrophoresis and matrix-assisted laser desorption/ionization with mass spectrometric detection.
\end{abstract}

Keywords: Metallothionein, dimerization, capillary electrophoresis, Förster resonance energy transfer

\section{INTRODUCTION}

Metallothioneins (MTs) are a family of low molecular weight proteins (6-7 kDa). MTs are involved in the metabolism of heavy metals and they serve as an intracellular reservoir of essential trace elements. In mammals they occur in four isoforms (MT-1 to MT-4) that vary in expression and localization in individual tissues. Each molecule consists of a single polypeptide chain of 61-68 amino acid residues with high cysteine content $(30 \%)$. The sulfhydryl group of cysteine express the most notable functional potential. It can form disulphide bonds by which MTs can create oligomers. Oligomers can be formed either under oxidative (e.g. presence of $\mathrm{NO}$ or hydrogen peroxide) or non-oxidative conditions (addition of excess of $\mathrm{Cd}^{2+}$ ). Investigation of their structural arrangement can help understand the development of oxidative stress and mechanism of transport of toxic metals. In addition, the oligomer formation may play an important role in a number of neurological disorders such as Alzheimer's or Parkinson's diseases [4,5]. Due to the MT characteristics, such as low molecular weight, unique primary structure with lack of aromatic amino acids, and numerous isoform occurrence analysis of MTs represent a demanding task in terms of satisfactory sensitivity and specificity. In addition to traditional analytical techniques, such as gel electrophoresis and liquid chromatography, capillary electrophoresis (CE) has proven to be a powerful separation method for MT analysis. CE belongs to those few analytical methods enabling the separation of single MT isoforms and their sensitive detection [1-3]. Matrixassisted laser desorption/ionization with mass spectrometric detection (MALDI MS) represents another effective tool for identification of the MT isoforms and/or MT oligomers.

Förster resonance energy transfer (FRET) is defined as a "long-range" dipole interaction between probes (the donor and the acceptor system) through resonance dipole-dipole coupling occurring in the pico- to nanometer range and thus at a scale relevant to changes in protein conformation [6]. Organic fluorophores such as cyanine dyes and quantum dots (QDs) are widely exploited as FRET probes for numerous chemical and biological applications such as protein folding investigation or for sensing and imaging of macromolecular interactions $[7,8]$. 
In the present work, the conjugation of MTs with QDs was investigated using CE coupled with absorbance detector and MALDI MS technique. We propose that the conjugation of protein to QDs could efficiently employed in FRET system which may subsequently serve for efficient investigation of MTs oligomerization.

\section{MATERIALS AND METHODS}

\subsection{Materials and reagents}

MT isoform (MT-1) from rabbit liver was obtained from Enzo Life Science, USA. Sodium borate, zinc acetate, cadmium acetate, sodium phosphate, mercaptosuccinic acid (MSA), isopropanol, $N$-Ethyl- $N$ '-(3dimethylaminopropyl)carbodiimide hydrochloride (EDC) and $\mathrm{N}$-Hydroxysulfosuccinimide sodium salt (SulfoNHS) were purchased from Sigma-Aldrich in ACS quality. The stock solution of MT (1 mg/mL) was prepared in ultrapure water and stored in the dark at $-20^{\circ} \mathrm{C}$. Ultrapure water purified by Milli-Q system was used for preparation of all solutions.

\subsection{Preparation of QDs}

Suspension of $\mathrm{ZnCd}$ QDs was prepared by mixing stock solutions of the $6 \mathrm{mM}$ zinc acetate, $6 \mathrm{mM}$ cadmium acetate, $20 \mathrm{mM}$ sodium phosphate buffer (PB) pH 7 and $16 \mathrm{mM}$ mercaptosuccinic acid (MSA). The resulting mixture with the typical molar ratio of 1:4:4:6 $\left(\mathrm{Cd}^{2+}: \mathrm{MSA}: \mathrm{PB}: \mathrm{Zn}^{2+}\right)$ was exposed to $U V$ irradiation for 5 minutes. The $\mathrm{ZnCd}$ QDs were precipitated by isopropanol and then isolated by centrifugation. Finally, ZnCd QDs were suspended in sodium phosphate buffer (PB, pH 7.2) and sonicated for 2 minutes before use.

\subsection{QDs bioconjugation}

At first, conjugation of ZnCd QDs with $0.2 \mathrm{mM} \mathrm{MT-1}$ was carried out by a method using coupling agents EDC and Sulfo-NHS according to the protocol published elsewhere [9].

\subsection{MT dimerization}

Dimers (or higher oligomers) of MTs were obtained by storing MT-1 $(0.1 \mathrm{mM})$ in $20 \mathrm{mM} \mathrm{PB}(\mathrm{pH} 9.2)$ in presence of different volumes $(0.5,1$ and $1.5 \mu \mathrm{L}) 0.5 \mathrm{mM} \mathrm{Cd}^{2+}$ at $8{ }^{\circ} \mathrm{C}$ under aerobic conditions for 1 week.

\subsection{Capillary electrophoresis}

Analyses of MTs conjugates were performed using CE 7100 (Agilent technologies, Waldbronn, Germany) equipped with UV/Vis absorbance detector. Fused silica capillary with an internal diameter of $75 \mu \mathrm{m}$, total length of $64.5 \mathrm{~cm}$ and effective length of $56 \mathrm{~cm}$ was used. The capillary was flushed with background electrolyte (BGE) for 15 minutes prior to the first use. The capillary was rinsed for $120 \mathrm{~s}$ with background electrolyte before each run to obtain stable measurements. Sample was injected hydrodynamically by pressure of $50 \mathrm{mbar}$ applied for $4 \mathrm{~s}$. Separation voltage was set to $20 \mathrm{kV}$. Sodium borate buffer $(20 \mathrm{mM}, \mathrm{pH} 9.2)$ was used as a BGE. The signal was registered at $254 \mathrm{~nm}$.

The method of "short-end injection" [10] was also introduced to be able to visualize the protein dimers/oligomers. Samples were injected at the end of the capillary near to the detector. Effective length of the capillary was $8.5 \mathrm{~cm}$. This was achieved by injecting the sample at the outlet end of the capillary (shortend injection), the capillary was air conditioned to temperature of $25^{\circ} \mathrm{C}$.

\subsection{MALDI-MS}

MT-1 was analysed using MALDI-TOF-MS (ultrafleXtreme instrument, Bruker Daltonik GmbH, Bremen Germany) equipped with a laser (operating at wavelength of $355 \mathrm{~nm}$ with an accelerating voltage of $25 \mathrm{kV}$, a 
maximum energy of $43.2 \mu \mathrm{J}$, and a repetition rate of $2000 \mathrm{~Hz}$ ) in linear positive ion mode. Organic matrix namely 2,5-Dihydroxybenzoic acid - was chosen. Matrix $(1 \mu \mathrm{L})$ was applied on the prepared sample of MT-1 with $\mathrm{Cd}^{2+}$ (probably dimers or higher oligomers) and dried under atmospheric pressure and ambient temperature $\left(25^{\circ} \mathrm{C}\right)$. The laser frequency was set to $1000 \mathrm{~Hz}$ and laser energy was optimized prior to each measurement. External calibration using a protein standard mixture I and II (Bruker Daltonics, Bremen, Germany) was applied in the range of $\mathrm{m} / \mathrm{z} 1-30 \mathrm{kDa}$. A total of 3000 spectra were summed for each spot using the Random Walk raster pattern, with no evaluation criteria and were analysed with the Flex Analysis software (Version 3.4).

\section{RESULTS AND DISCUSSION}

A solution of metallothionein isoform MT-1 at millimolar concentrations stored under aerobic conditions at $8{ }^{\circ} \mathrm{C}$ per 1 week produces a new species characterized here as dimers and/or higher oligomers. Here we confirmed that the environment has a great influence on the oxidation rate. Non-oxidative dimerization caused by $\mathrm{Cd}^{2+}$ addition occurred and metal bridges were formed. Samples of MTs with $\mathrm{Cd}^{2+}$ were analysed by CE. The obtained electropherograms are shown in Figure 1, where distinct changes in the signal intensities are observed. These changes suggest that the $\mathrm{Cd}^{2+}$ addition causes significant changes in the MTs structure.

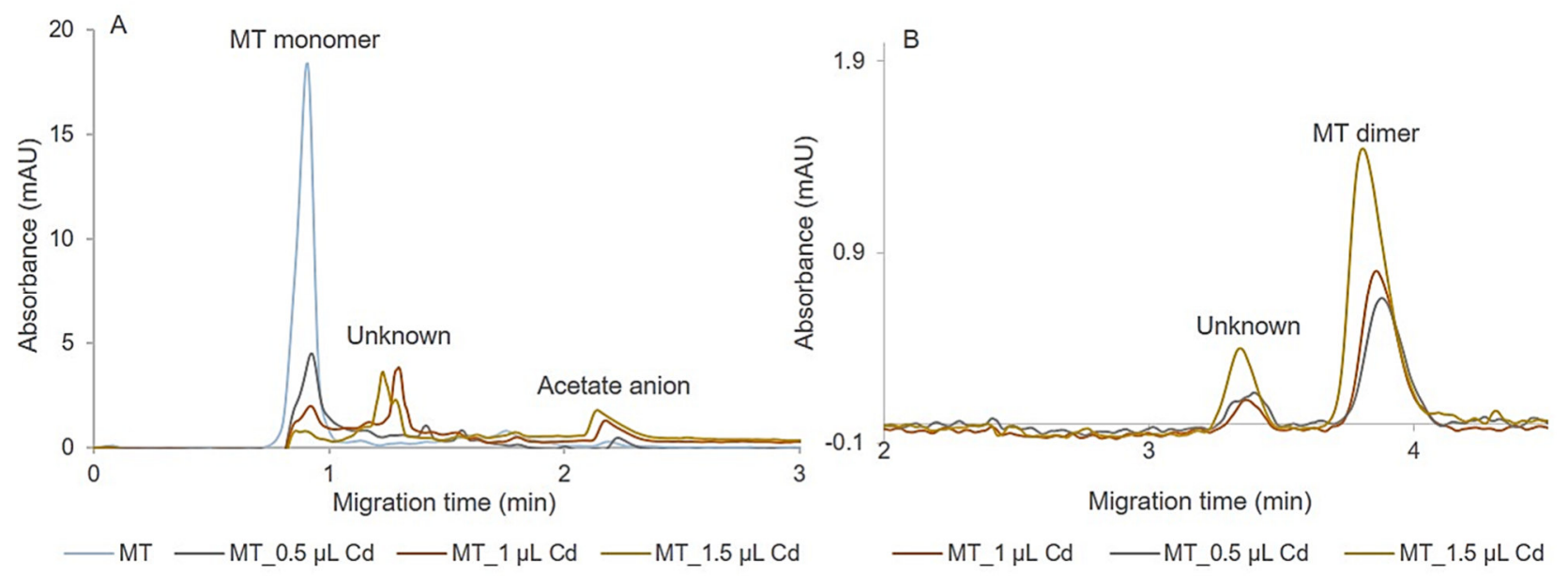

Figure 1 Electropherograms of metallothionein with different volumes $(0.5,1$ and $1.5 \mu \mathrm{L})$ of $0.05 \mathrm{mM} \mathrm{Cd}^{2+}$, at positive polarity (A) and negative polarity (B) by short-end injection.

The short end injection method was used to demonstrate the dimerization of MT-1. As shown in the Figure $1 \mathrm{~A}$, with the increased amount of $\mathrm{Cd}^{2+}$, the peak of MT-1 monomer with migration time of 0.9 minute decreased (Figure 1A), while the peak of MT-1 dimer with migration time 3.8 minutes increased (Figure 1B). Based on these results we hypothesise that the highest volume of $\mathrm{Cd}^{2+}\left(1.5 \mu \mathrm{L} 0.05 \mathrm{mM} \mathrm{Cd}^{2+}\right)$ directly affects the formation of metal bridges in the investigated protein MT-1 causing a subsequent dimerization of MTs. MALDI-TOF-MS analysis of the identical samples confirmed the formation of dimers increased after addition of $1.5 \mu \mathrm{L}$ of $0.05 \mathrm{mM} \mathrm{Cd}^{2+}$ to the MT-1 sample and one-week storage at the $8{ }^{\circ} \mathrm{C}$ under aerobic conditions (Figure 2).

Even though the CE and MALDI-TOF-MS were able to detect and determine the formation of MT dimers/oligomers, these methods do not provide any information about the structure of the formed species. For this reason, we suggest the use of FRET technique for following investigation. At first the FRET pair composed of $\mathrm{ZnCd} \mathrm{QD}$ and cyanine dye was proposed. 


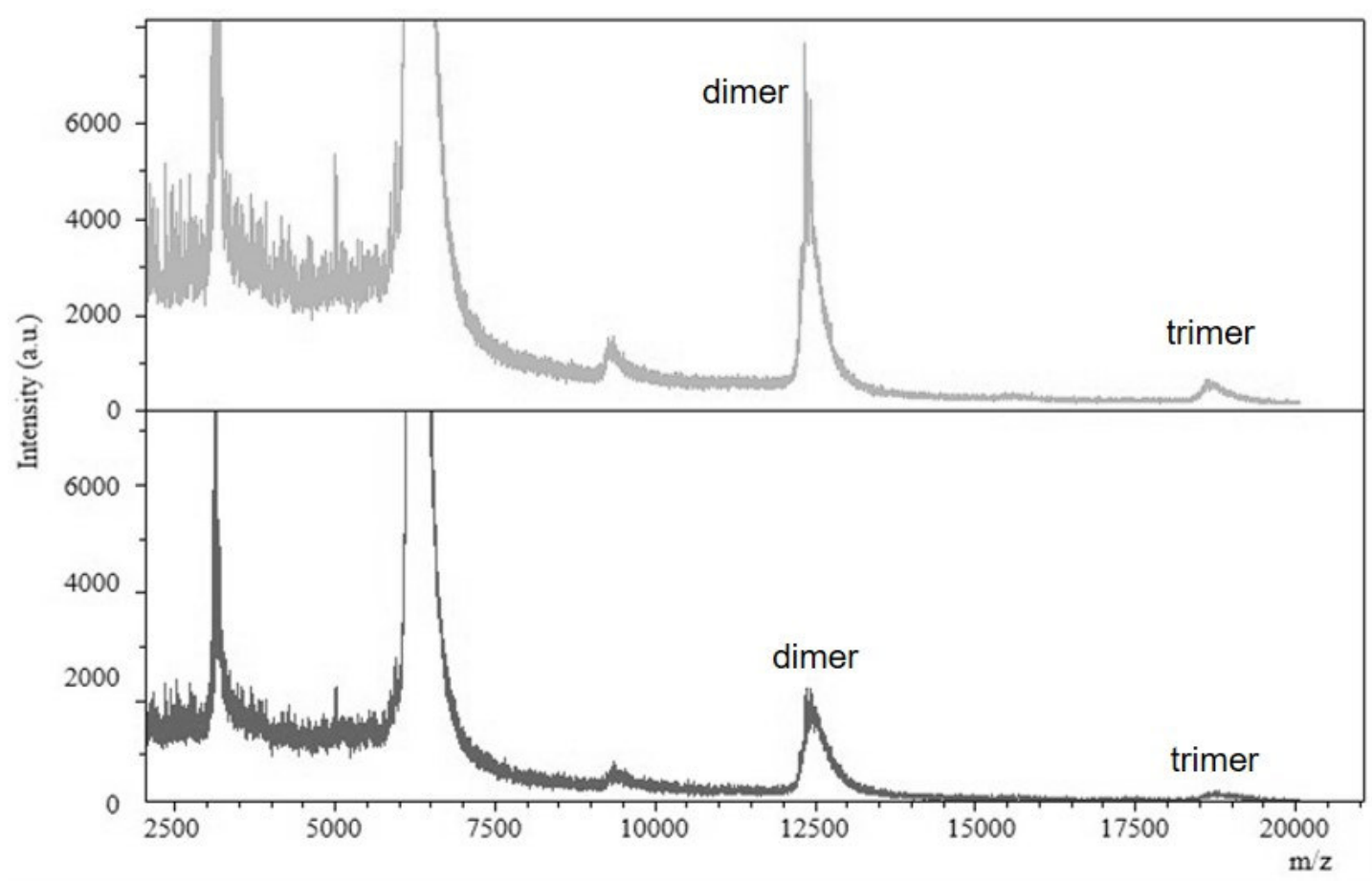

Figure 2 MALDI-TOF-MS spectra of MTs. Sample of MTs without $\mathrm{Cd}^{2+}$ addition analysed immediately (bottom) and sample of MTs with $\mathrm{Cd}^{2+}$ addition after 1 week-storage (top)

At first, the conjugation of MTs with quantum dots (QDs) was studied in order to evaluate the ability of the FRET system to indicate the structure of MT dimers or oligomers. However, the short-end injection method did not provide sufficient separation resolution. Therefore CE method with the effective length of the capillary of $56 \mathrm{~cm}$ was used resulting in successful separation of MT, QDs and their conjugates (Figure 3). The conjugation of MTs with organic fluorophores will be subject of further research aiming to study a protein oligomerization by FRET.

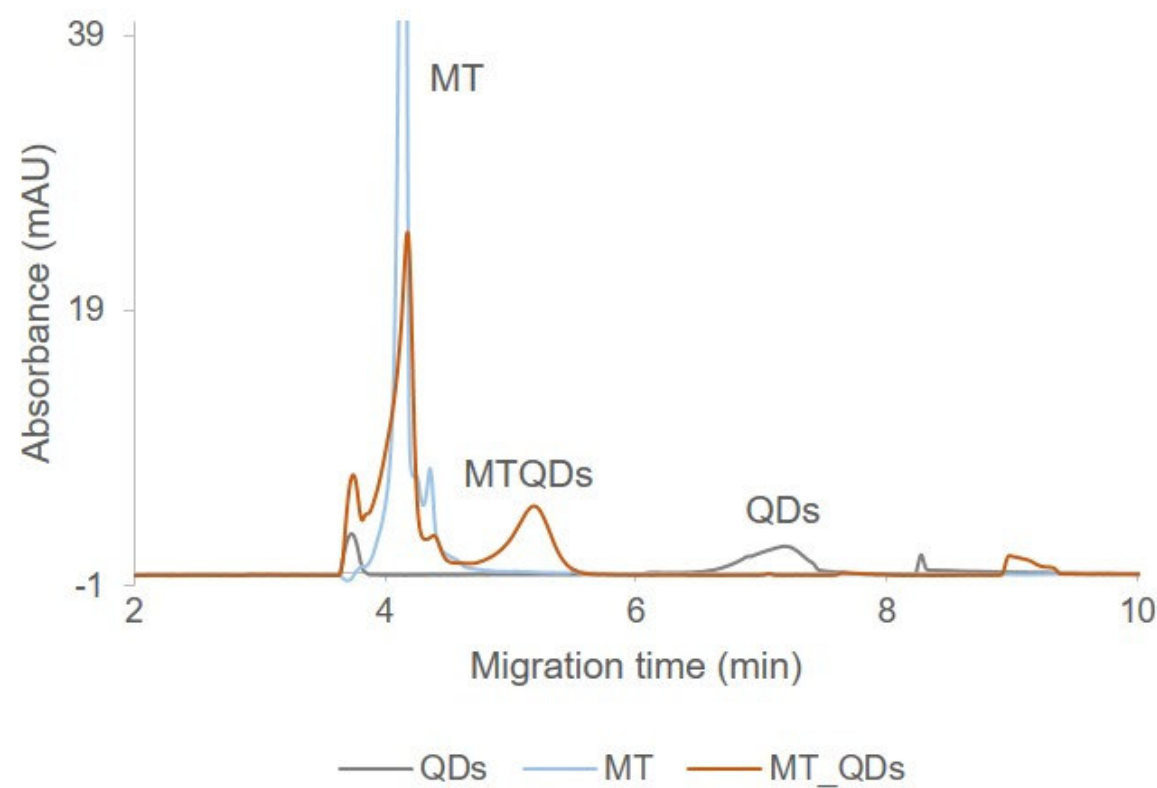

Figure 3 Electropherograms of protein (MT-1) conjugation to quantum dots 


\section{CONCLUSION}

Metallothionein (MT) may form dimers (as well as higher oligomers) upon storage under aerobic conditions. This dimerization may play an essential role as a mediator in oxidoreduction signalling pathways. In this study, the oligomerization of MT was investigated using capillary electrophoresis coupled to absorbance detection and mass spectrometry. Dimers/higher oligomers of MT were studied by a CE and verified by a MALDI-TOFMS analysis after $\mathrm{Cd}^{2+}$ addition and 1 week-storage. The method of protein conjugation to QDs was employed in order to investigate the FRET system applicability for structural characterization of protein dimerization. Generally, fluorescence-based detection techniques are very sensitive as well as highly selective with high potential for multiplexing. Moreover, Förster resonance energy transfer enables the measurements of distances at the molecular level and monitor distance changes in nanometer range. This phenomenon is highly dependent on the distance between acceptor and donor fluorophores. In conclusion, analysis based on a combination of CE FRET could be a very useful tool for study of protein oligomerization due to low cost and ease of use.

\section{ACKNOWLEDGEMENTS}

\section{The research was financially supported by Internal Grant Agency MENDELU IGA grant, no. IP_025/2019.}

\section{REFERENCES}

[1] KEPINSKA, M. and H. MILNEROWICZ. Capillary electrophoresis of metallothionein. Vol. 2. 2015.

[2] SANZ-NEBOT, V., B. ANDÓN, and J. BARBOSA. Characterization of metallothionein isoforms from rabbit liver by liquid chromatography coupled to electrospray mass spectrometry. Journal of Chromatography B, 2003. 796(2): p. 379-393.

[3] RYVOLOVA, M., et al. Structural changes in metallothionein isoforms revealed by capillary electrophoresis and Brdicka reaction. Electrophoresis, 2012. 33: p. 270-9.

[4] ZANGGER, K., et al. Oxidative dimerization in metallothionein is a result of intermolecular disulphide bonds between cysteines in the alpha-domain. Biochemical Journal, 2001. 359: p. 353-360.

[5] KASSIM, R., C. RAMSEYER, and M. ENESCU. Oxidation reactivity of zinc-cysteine clusters in metallothionein. Journal of biological inorganic chemistry: JBIC: a publication of the Society of Biological Inorganic Chemistry, 2013. 18.

[6] OKUMOTO, S., H. TAKANAGA, and W. FROMMER. Quantitative imaging for discovery and assembly of the metabo-regulome: Tansley review. The New phytologist, 2008. 180: p. 271-95.

[7] LI, Y.-Q., et al. High-sensitivity quantum dot-based fluorescence resonance energy transfer bioanalysis by capillary electrophoresis. Biosensors and Bioelectronics, 2010. 25(6): p. 1283-1289.

[8] BUCKHOUT-WHITE, S., et al. Assembling programmable FRET-based photonic networks using designer DNA scaffolds. Nature communications, 2014. 5: p. 5615.

[9] PEREIRA, M. and E. LAI. Capillary Electrophoresis for the Characterization of Quantum Dots After Non-Selective or Selective Bioconjugation with Antibodies for Immunoassay. Journal of nanobiotechnology, 2008. 6: p. 10.

[10] FABRE, H. and N. MESPLET. Robustness testing for a capillary electrophoresis method using the "short-end injection" technique. Journal of chromatography A, 2000. 897: p. 329-38. 